\title{
On the category of Euclidean configuration spaces and associated fibrations
}

\author{
FRIDOLIN ROTH
}

\begin{abstract}
We calculate the Lusternik-Schnirelmann category of the $k^{\text {th }}$ ordered configuration spaces $F\left(\mathbb{R}^{n}, k\right)$ of $\mathbb{R}^{n}$ and give bounds for the category of the corresponding unordered configuration spaces $B\left(\mathbb{R}^{n}, k\right)$ and the sectional category of the fibrations $\pi_{k}^{n}: F\left(\mathbb{R}^{n}, k\right) \rightarrow B\left(\mathbb{R}^{n}, k\right)$. We show that secat $\left(\pi_{k}^{n}\right)$ can be expressed in terms of subspace category. In many cases, eg, if $n$ is a power of 2, we determine cat $\left(B\left(\mathbb{R}^{n}, k\right)\right)$ and $\operatorname{secat}\left(\pi_{k}^{n}\right)$ precisely.

$55 \mathrm{M} 30 ; 55 \mathrm{R} 80,55 \mathrm{~S} 40$
\end{abstract}

\section{Motivation and results}

The sectional category $\operatorname{secat}(p)$ of a fibration $p: E \rightarrow B$ is defined to be the least integer $n$ such that the base $B$ can be covered with $n+1$ open sets admitting local sections (Cornea, Lupton, Oprea, and Tanré [9, 9.13]). This notion, as in Švarc [22, page 70] sometimes defined without the +1 -shift and referred to as the Schwarz genus of $p$, has proved very useful. For instance, consider the fibration $\pi_{k}^{2}: F\left(\mathbb{R}^{2}, k\right) \rightarrow B\left(\mathbb{R}^{2}, k\right)$ from the ordered configuration space $F\left(\mathbb{R}^{2}, k\right)=\left\{\left(x_{1}, x_{2}, \ldots, x_{k}\right) \in\left(\mathbb{R}^{2}\right)^{k} \mid x_{i} \neq x_{j}\right.$ for $i \neq$ $j\}$ to its unordered quotient $B\left(\mathbb{R}^{2}, k\right):=F\left(\mathbb{R}^{2}, k\right) / \Sigma_{k}$ given by the obvious action of the symmetric group $\Sigma_{k}$ on $k$ letters. The sectional category of the fibration $\pi_{k}^{2}: F\left(\mathbb{R}^{2}, k\right) \rightarrow B\left(\mathbb{R}^{2}, k\right)$ has attracted some attention since Smale showed that it provides a lower bound for the complexity of algorithms computing the (pairwise disjoint) roots of a complex polynomial of degree $k$ (Smale [21], Vasiliev [24; 23], de Concini, Procesi and Salvetti [10] and Arone [2]). Nevertheless secat $\left(\pi_{k}^{2}\right)$ has not yet been determined for all $k$.

We now recollect what is known on $\operatorname{secat}\left(\pi_{k}^{2}\right)$ so far: Vassiliev showed the inequality $k-D_{p}(k) \leq \operatorname{secat}\left(\pi_{k}^{2}\right) \leq k-1$ where $D_{p}(k)$ is the sum of the coefficients in the $p$-adic extension of $k[24 ; 23]$. In particular, this gives $\operatorname{secat}\left(\pi_{k}^{2}\right)=k-1$ if $k$ is a power of a prime. If $k$ is not a power of a prime, it is very difficult to determine $\operatorname{secat}\left(\pi_{k}^{2}\right)$ precisely, and it is only recently that some progress has been made. In [10] de Concini, Procesi and Salvetti developed an obstruction theory to decide whether 
$\operatorname{secat}\left(\pi_{k}^{2}\right)$ equals its known upper bound $k-1$ and showed that for $k=6-$ the least $k$ for which the question was open - in fact it does not. Based on that theory, Gregory Arone did some more calculations and showed that $\operatorname{secat}\left(\pi_{k}^{2}\right)<k-1$ holds for all $k$ which are neither the power of a prime nor twice the power of a prime [2]. If $k=2 p^{\ell}$, the question whether $\operatorname{secat}\left(\pi_{k}^{2}\right)=k-1$ is still open for some $\ell$ and odd $p$, as well as the precise determination of $\operatorname{secat}\left(\pi_{k}^{2}\right)$ in many other cases.

In this paper we begin to study the sectional category $\operatorname{secat}\left(\pi_{k}^{n}\right)$ of the fibrations $\pi_{k}^{n}: F\left(\mathbb{R}^{n}, k\right) \rightarrow B\left(\mathbb{R}^{n}, k\right)$ for varying $n \in \mathbb{N}$. This is closely related to the LusternikSchnirelman category cat $\left(B\left(\mathbb{R}^{n}, k\right)\right)$ of the unordered configuration spaces $B\left(\mathbb{R}^{n}, k\right)$. Here we follow [9] and say that the Lusternik-Schnirelman category cat $(X)$ of a topological space $X$ is the least integer $m$ such that $X$ can be covered with $m+1$ open sets, which are all contractible within $X$. One elementary relation between $\operatorname{secat}\left(\pi_{k}^{n}\right)$ and $\operatorname{cat}\left(B\left(\mathbb{R}^{n}, k\right)\right)$ is the general fact that the sectional category of a fibration is bounded above by the category of its base. Together with $\operatorname{cat}\left(B\left(\mathbb{R}^{n}, k\right)\right) \leq(k-1) \cdot(n-1)$ (Lemma 4.1) this gives an upper bound for $\operatorname{secat}\left(\pi_{k}^{n}\right)$. Moreover, in our cases, we get descriptions of $\operatorname{secat}\left(\pi_{k}^{n}\right)$ in terms of the category of a map and subspace category, definitions whereof are given in Section 2:

Theorem 1.1 Let $n, k, r \in\{1,2,3, \ldots\}$ and let $\pi_{k}^{n}: F\left(\mathbb{R}^{n}, k\right) \rightarrow B\left(\mathbb{R}^{n}, k\right)$ be the obvious fibration. More generally we can admit $r=\infty$ as well. Then

$$
\operatorname{secat}\left(\pi_{k}^{n}\right)=\operatorname{cat}\left(B\left(\mathbb{R}^{n}, k\right) \hookrightarrow B\left(\mathbb{R}^{n+r}, k\right)\right)=\operatorname{cat}_{B\left(\mathbb{R}^{n+r}, k\right)} B\left(\mathbb{R}^{n}, k\right) .
$$

The key to this observation is to consider the ordered Euclidean configuration spaces as well. Another reason for considering the spaces $F\left(\mathbb{R}^{n}, k\right)$ is that cat $\left(F\left(\mathbb{R}^{n}, k\right)\right)$ gives a lower bound for $\operatorname{cat}\left(B\left(\mathbb{R}^{n}, k\right)\right)$ by the usual covering argument. In general this bound is quite bad. For $n=2$ however, it allows to precisely determine cat $\left(B\left(\mathbb{R}^{2}, k\right)\right)$ and shows that the subtleties in the calculation for $\sec a t\left(\pi_{k}^{2}\right)$ do not arise in the calculation of $\operatorname{cat}\left(B\left(\mathbb{R}^{2}, k\right)\right)$, which turns out to be $k-1$ for all $k$. The following result might also be of interest for its own sake.

Theorem 1.2 For all $n \geq 1$, ie, as long as $F\left(\mathbb{R}^{n+1}, k\right)$ is connected,

$$
\operatorname{cat}\left(F\left(\mathbb{R}^{n+1}, k\right)\right)=k-1 .
$$

The space $F(\mathbb{R}, k)$ consists of $k$ ! contractible components, hence

$$
\operatorname{cat}(F(\mathbb{R}, k))=k !-1 .
$$


It would be nice to have an analogous statement for the category of the unordered Euclidean configuration spaces, able to compete with the previous theorem in simplicity. We hold the following quite plausible:

Conjecture 1.3 For all $n$ and $k$

$$
\operatorname{cat}\left(B\left(\mathbb{R}^{n}, k\right)\right)=(k-1) \cdot(n-1) .
$$

Among other indications, our optimism is based on the following calculations. We use the more usual notation $\alpha(k)$ for $D_{2}(k)$ :

Theorem 1.4 Let $\alpha(k)=D_{2}(k)$ be the number of 1's in the dyadic expansion of $k$. Then we have

$$
(k-\alpha(k)) \cdot(n-1) \leq \operatorname{secat}\left(\pi_{k}^{n}\right) \leq \operatorname{cat}\left(B\left(\mathbb{R}^{n}, k\right)\right) \leq(k-1) \cdot(n-1) .
$$

Theorem 1.5 In case $k$ is a power of 2 or $k=3$ or if $n$ is odd and $k=p$ is any prime, we have

$$
\operatorname{secat}\left(\pi_{k}^{n}\right)=\operatorname{cat}\left(B\left(\mathbb{R}^{n}, k\right)\right)=(k-1) \cdot(n-1) .
$$

Moreover, for any $k$ we have

$$
\operatorname{cat}\left(B\left(\mathbb{R}^{2}, k\right)\right)=(k-1) .
$$

These results are obtained by exploiting work of Vassiliev, cohomology calculations by Fred Cohen and combining them with standard results from LS-theory and the concept of category weight. The statement for $k=3$ follows together with Theorem 1.1 and geometric insight. The general upper bound in Theorem 1.4 can be derived from the following lemma, which we could not find in literature.

Lemma 1.6 Let $X$ be an $n$-dimensional CW-complex, $X^{(r)}$ its $r$-skeleton and assume that $X-X^{(k-1)}$ is connected. Then cat $\left(X-X^{(k-1)}\right) \leq n-k$.

We are aware of the incompleteness of Theorem 1.4. We are also aware that improvements can be achieved. For instance, we gained some generalizations in [20]. However, we did not obtain a complete generalization of Vassiliev's results, new improvements of the upper bound for $\operatorname{secat}\left(\pi_{k}^{n}\right)$, an unbounded sequence $\left(n_{i}\right)$ such that $\operatorname{cat}\left(B\left(\mathbb{R}^{n_{i}}, k\right)\right)=(k-1)\left(n_{i}-1\right)$ for all $k$ or of course a proof or disproof of Conjecture 1.3.

We think it is worth mentioning that the behaviour showing up in Vassiliev's calculations and our Theorem 1.4, as well as the lack of complete information seem not to be unusual. 
For example, take the immersion problem for real projective spaces $\mathbb{R} P^{n}$ into $\mathbb{R}^{m}$ which is still open in the general case. Whitney's embedding theorem says that an immersion exists at least for $m$ greater or equal to $2 n-1$. This bound is taken if $n$ is a power of 2 . More generally, the number $\alpha(n)$ appears in Ralph Cohen's general immersion theorem which says that every compact, differentiable, $n$-dimensional manifold immerses in Euclidean space of dimension $2 n-\alpha(n)$ [8]. For the case of complex projective spaces it has been conjectured, that the immersion dimension is $4 n-2 \alpha(n)+\epsilon$, where $\epsilon$ is a non-negative integer bounded roughly by 3 , see González [15] and references therein. In fact, these immersion problems are closely related to invariants of the category type. For instance, in the real case and $n \neq 1,3,7$, Faber, Tabachnikov and Yuzvinsky showed in [13] that the immersion dimension is the sectional category of $P \mathbb{R} P^{n} \rightarrow \mathbb{R} P^{n} \times \mathbb{R} P^{n}$, up to shift also known as the topological complexity of $\mathbb{R} P^{n}$. Here $P \mathbb{R} P^{n}$ is the space of all continuous paths $\gamma:[0,1] \rightarrow \mathbb{R} P^{n}$, and the fibration is evaluation at the end points. This notion was also useful for the immersion problem for $2^{e}$-torsion lens-spaces for $e>1$ as an approach to the immersion problem for complex projective spaces [15].

As another example consider the Lusternik-Schirelman category of the real Grassmann manifolds $G_{n, k}$ of $k$ dimensional subspaces in $\mathbb{R}^{n+k}$. By dimensional reasons cat $\left(G_{n, k}\right) \leq n k$ and Berstein showed in [3], that this bound is taken if and only if $n=1$ or $k=1$ or $\left(n=2\right.$ and $\left.k=2^{m}-1\right)$ or $\left(k=2\right.$ and $\left.n=2^{m}-1\right)$. We are not aware of precise determinations in the general case.

This paper developed from the author's diploma thesis [20] which is more detailed in a number of points. However, here we put more emphasis on the sectional category point of view.

Acknowledgements First of all I would like to thank my advisor Carl-Friedrich Bödigheimer, who suggested to investigate the LS-category of configuration spaces and guided my work which lead to [20]. I am also very grateful to Fred Cohen whom I met first when I started this project and attended his lectures in Louvain-la-Neuve. Besides its mathematical interest, his comments and encouraging remarks were of invaluable mental support. I like to thank the organizers for the wonderful conference and the invitation to Tokyo. I'm also grateful to Daniel Tanré, Sadok Kallel and Yves Félix whom I could talk to in Lille about the subject. Jesús González drew my attention to the close relation between sectional category and immersion questions. Special thanks are to Birgit Richter for critical remarks, corrections and discussion of the manuscript. Last but not least I'd like to thank the German National Academic Foundation for their support. 


\section{Lusternik-Schnirelman category}

The investigation of numerical homotopy invariants called category began with an article by Lusternik and Schnirelman [18]. Their aim was to obtain bounds for the number of critical points of a smooth function on a manifold. Since then various slightly differing definitions showed up in the literature. The definition given in the introduction takes into account that we should have $\operatorname{cat}(*)=0$ for a point or contractible space $*$. We followed [9] and also recommend this book as a source for the following results.

We now give a unifying approach to various notions of category including those mentioned above. For that purpose we define the category cat $(f)$ of a map $f: A \rightarrow B$. Let cat $(f)$ be the least integer $n$, such that $A$ can be covered with $n+1$ open sets and the restriction of $f$ to each of these sets is nullhomotopic. Such a cover of $A$ is called categorical.

We recover the definitions of the introduction via $\operatorname{cat}(X)=\operatorname{cat}\left(i d_{X}\right)$ and also secat $(p)$ equals cat $\left(f_{p}\right)$ when $f_{p}$ is a classifying map for a principal fibration $p$, see $[9,9.18$, 9.19] and Hatcher [16, Exercise 22 page 420]. We will only deal with fibrations of this type and can hence use $\operatorname{secat}(p)$ and $\operatorname{cat}\left(f_{p}\right)$ interchangeably in the sequel. Furthermore, for $A \subset B$ the subspace category of $\operatorname{cat}_{B}(A)$ is defined to be the least $n$ such that there exists a cover of $A$ with $n+1$ subsets of $B$, each open and contractible in $B$. For an open inclusion $i: A \hookrightarrow B$ one obviously has $\operatorname{cat}_{B}(A)=\operatorname{cat}(i)$.

Alternative definitions for $\operatorname{cat}(X)$ which agree with the standard one under mild hypotheses, including the case where $X$ is a pointed CW-complex, have been given by Whitehead and Ganea. For a space $X$ Ganea constructed a sequence of fibrations $p_{n}: G_{n}(X) \rightarrow X$ which have a section if and only if $\operatorname{cat}(X) \leq n$.

We now recollect some properties of the category of a space $X$ :

\section{Proposition 2.1}

(1) If $X$ dominates $Y$, ie, if there are maps $f: X \rightarrow Y$ and $g: Y \rightarrow X$ such that $f \circ g \simeq i d_{Y}$, then cat $Y \leq$ cat $X$. In particular, category is a homotopy invariant.

(2) We have $\operatorname{cat}(E) \leq \operatorname{cat}(B)$ for a covering $p: E \rightarrow B$ with $E$ path-connected.

(3) We have $\operatorname{secat}(p) \leq \operatorname{cat}(B)$ for a fibration $p: E \rightarrow B$.

(4) We have $\operatorname{cup}_{R}(X) \leq \operatorname{cat}(X)$ where $\operatorname{cup}_{R}(X)$ is the $R$-cuplength for any coefficient ring $R$, ie, the least $n$ such that all cup-products of at least $n+1$ non-trivial factors in $\tilde{H}^{*}(X ; R)$ vanish.

(5) If $X$ is an $(n-1)$-connected $C W$-complex, then $\operatorname{cat}(X) \leq \frac{\operatorname{dim}(X)}{n}$. 
All proofs are elementary and can be found in [9, Chapters 1 and 3]. Since the statement of Lemma 1.6 fits very well in this collection, we now give its proof:

Proof of Lemma 1.6 The idea is the same as one can use to show that every CWsubcomplex is a strong deformation-retract of some open neighborhood. First note that subadditivity (ie, cat $_{X}(A \cup B) \leq \operatorname{cat}_{X}(A)+\operatorname{cat}_{X}(B)+1$ for $\left.A, B \subset X\right)$ yields

$$
\begin{aligned}
\operatorname{cat}\left(X-X^{(k-1)}\right) & =\operatorname{cat}_{X-X^{(k-1)}}\left(\coprod_{r=k}^{n} X^{(r)}-X^{(r-1)}\right) \\
& \leq n-k+\sum_{r=k}^{n} \operatorname{cat}_{X-X^{(k-1)}}\left(X^{(r)}-X^{(r-1)}\right) .
\end{aligned}
$$

Hence it suffices to show that cat $X-X^{(k-1)}\left(X^{(r)}-X^{(r-1)}\right)=0$ for all $r \in\{k, k+$ $1, \ldots, n\}$, ie, that $X^{(r)}-X^{(r-1)}$ is covered by some set which is open and contractible in $X-X^{(k-1)}$. Fix $r \in\{k, k+1, \ldots, n\}$ for the sequel. $X^{(r)}-X^{(r-1)}$ is a disjoint union of $r$-balls, hence contractible in $X-X^{(k-1)}$, since $X-X^{(k-1)}$ is pathconnected. We are left to show that $X^{(r)}-X^{(r-1)}$ is a retract of some set $V_{n}$, open in $X-X^{(k-1)}$. The requirement that $V_{n}$ is contractible in the ambient space $X-X^{(k-1)}$ is then automatically satisfied since $X^{(r)}-X^{(r-1)}$ is a disjoint union of $r$-cells, hence contractible in the ambient space: Each of the cells is contractible to a point and then we use that in our case the ambient space is path-connected.

We will obtain $V_{n}$ by recursively defining sets $V_{\ell}$ for $r \leq \ell \leq n$, such that $V_{\ell} \supset$ $X^{(r)}-X^{(r-1)}$ is open in $X^{(\ell)}-X^{(k-1)}$ and retracts to $X^{(r)}-X^{(r-1)}$ : For $V_{r}$ we can just take $V_{r}:=X^{(r)}-X^{(r-1)}$. If $V_{\ell} \supset X^{(r)}-X^{(r-1)}$ as required is already defined, extend it to obtain $V_{\ell+1}$ as follows: For each $(\ell+1)$-cell $e$ choose a point $x_{e}$ in its interior, as well as radial homotopies $h_{t}^{e}: X^{(\ell)} \cup\left(e-x_{e}\right) \rightarrow X^{(\ell)} \cup\left(e-x_{e}\right)$, ie, homotopies relative $X^{(\ell)}$ with $h_{0}^{e}=i d, h_{1}^{e}: X^{(\ell)} \cup\left(e-x_{e}\right) \rightarrow X^{(\ell)}$ and $h_{1}^{e} \circ h_{t}^{e}=h_{1}^{e}$. The set $\left(h_{1}^{e}\right)^{-1}\left(V_{\ell}\right)$ then is obtained from $V_{\ell}$ by glueing a truncated cone over $V_{\ell} \cap \partial e$, open in $\bar{e}$. Define $V_{\ell+1}:=\bigcup_{e}\left(h_{1}^{e}\right)^{-1}\left(V_{\ell}\right)$, taking the union over all $(\ell+1)$-cells $e$. The set $V_{\ell+1} \supset X^{(r)}-X^{(r-1)}$ then is open in $X^{(\ell+1)}-X^{(k-1)}$ (weak topology) and retractible to $X^{(r)}-X^{(r-1)}$. This shows cat $X-X^{(k-1)}\left(X^{(r)}-X^{(r-1)}\right)=0$.

Most of our lower bounds for the sectional category are obtained through the concept of

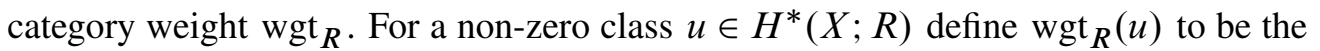
greatest $k$ (or $\infty)$, such that $p_{k-1}^{*}(u)=0 \in H^{*}\left(G_{k-1}(X) ; R\right)$ for the $(k-1)$ st Ganea fibration $p_{k-1}: G_{k-1}(X) \rightarrow X$. We recollect important properties and consequences from [9, pages 63f, 242ff and 261f]. The last point is a consequence of [9, Proposition 9.18 and 8.22(2)]: 
Proposition 2.2 Let $u \in H^{k}(X ; R)$ be non-zero. Then:

(1) If $u \in H^{k}(K(\pi, 1) ; R)$ is a class in the cohomology of an Eilenberg-MacLane space of type $(\pi, 1)$, then $\operatorname{wgt}_{R}(u)=k$.

(2) If $f: Y \rightarrow X$ is such that $f^{*}(u) \neq 0$, then $\operatorname{wgt}_{R}\left(f^{*}(u)\right) \geq \operatorname{wgt}_{R}(u)$. In other words: If a cohomology class does not vanish under pullback, then its category weight cannot decrease.

(3) If $p: E \rightarrow Y$ is a fibration arising as a pullback over $f: Y \rightarrow X$ of a fibration $\widehat{E} \rightarrow X$ with contractible total space $\widehat{E}$ and $f^{*}(u) \neq 0$, then $\operatorname{wgt}_{R}(u) \leq$ $\operatorname{secat}(p)$

\section{Cellular models, geometry and cohomology of Euclidean configuration spaces}

In this section we will collect the necessary algebraic and geometric data in order to derive bounds for the category of Euclidean configuration spaces in combination with the results of Section 2. The maps and spaces under consideration fit into the following fundamental diagram

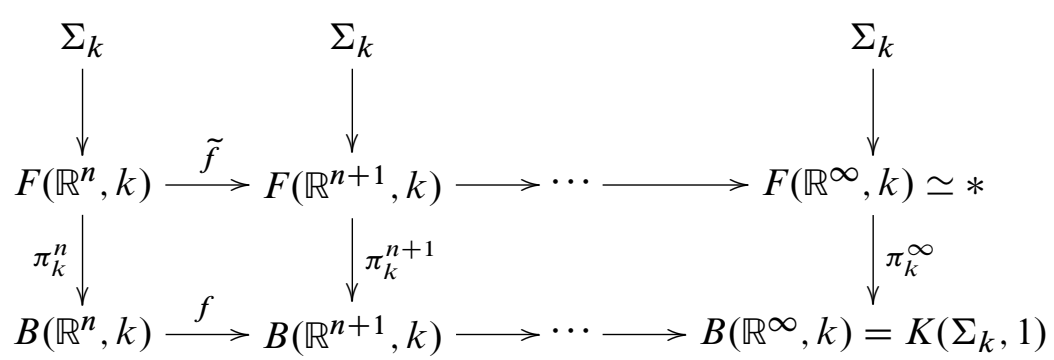

where the vertical maps are the coverings given by the free action of the symmetric group $\Sigma_{k}$ and horizontal maps are induced by the inclusion of $\mathbb{R}^{n}$ into $\mathbb{R}^{n+1}$. It follows from Fadell's and Neuwirth's fundamental sequence of fibrations [12], that $F\left(\mathbb{R}^{n}, k\right)$ is $n-2$ connected. As a consequence the limit spaces on the right give the universal covering of an Eilenberg-MacLane space $K\left(\Sigma_{k}, 1\right)$. Note for later use that all the rectangles in diagram (1) are homotopy pullbacks.

The integer cohomology of Euclidean configuration spaces was calculated by Fred Cohen [6; 7]. In the formulation of [5] and for $n \geq 2, H^{*}\left(F\left(\mathbb{R}^{n}, k\right)\right)$ is given by generators $A_{i, j},(1 \leq j<i \leq k)$ all in degree $n-1$, subject to the relations 
(1) $A_{i, j}^{2}=0$

(2) $A_{i, j} A_{i, \ell}=A_{\ell, j}\left(A_{i, \ell}-A_{i, j}\right)$ for $j<\ell<i$

(3) associativity and graded commutativity.

We can draw the following conclusions:

Corollary 3.1 For all $n \geq 2$, we have $\operatorname{cup}_{\mathbb{Z}}\left(F\left(\mathbb{R}^{n}, k\right)\right)=k-1$.

Corollary 3.2 Let $n \geq 3$. Then $F\left(\mathbb{R}^{n}, k\right)$ is homotopy equivalent to a $C W$-complex $Y$ with cells just in dimension $q \cdot(n-1)$ for $q \in\{0,1, \ldots, k-1\}$.

Proof Follow the construction in [16, 4.C page 429] and note that in our case the homology is free. A geometric construction (also for $n=2$ ) can also be found in [11, Sections VI.8,VI.10].

These corollaries will allow to calculate the category of ordered configuration spaces completely and we now turn to the unordered case and sectional category.

We are going to apply the concept of category weight using the fundamental diagram (1). It turns out that we can draw a lot of information from a CW-decomposition of the one-point compactification $B\left(\mathbb{R}^{n}, k\right)_{\infty}$ of $B\left(\mathbb{R}^{n}, k\right)$ introduced by Vassiliev [24][23, pages 28ff]. This decomposition is a generalization of the one introduced by Fuks for $n=2$ in [14]. Vassiliev describes the various cells of his model as well as their boundaries mod 2 in terms of certain so-called $(n, k)$-trees. A precise description of what an $(n, k)$-tree looks like is given in the construction [23, page 28]. We only note that an $(n, k)$-tree has at least $k+n-1$ and at most $k \cdot n$ edges. Vassiliev has proven that for any $n, k$ there exists the structure of a $C W$-complex of the space $B\left(\mathbb{R}^{n}, k\right)_{\infty}$ with cells being sets of points corresponding to various $(n, k)$-trees and the added point [23, lemma 3.3.1, page 29]. Furthermore, the dimension of such a cell is equal to the number of edges in the corresponding tree [23, lemma 3.3.2, page 29]. Altogether, this leads to the following observation:

Proposition 3.3 (Vassiliev) There is a $C W$-decomposition of the one-point compactification $B\left(\mathbb{R}^{n}, k\right)_{\infty}$ having the point $\infty$ as the only cell of dimension 0 . All other cells have dimension $r$ with $k+n-1 \leq r \leq k \cdot n$.

There is a stabilization of these models as $n$ turns to $\infty$, and using Poincaré-Lefschetz duality, Vassiliev shows: 
Proposition 3.4 [23, page 27] The homomorphism

$$
H^{*}\left(B\left(\mathbb{R}^{\infty}, k\right) ; \mathbb{Z} / 2 \mathbb{Z}\right) \rightarrow H^{*}\left(B\left(\mathbb{R}^{n}, k\right) ; \mathbb{Z} / 2 \mathbb{Z}\right)
$$

induced by the map from the fundamental diagram (1) is surjective.

The reader who is familiar with Vassiliev's cell decomposition and its description in terms of trees may also derive the following:

Corollary 3.5 Let $\alpha(k)$ be the number of 1 's in the dyadic decomposition of $k$. Then

$$
H^{q}\left(B\left(\mathbb{R}^{n}, k\right) ; \mathbb{Z} / 2 \mathbb{Z}\right) \begin{cases}=0 & \text { if } q>(k-\alpha(k)) \cdot(n-1) \\ \neq 0 & \text { if } q=(k-\alpha(k)) \cdot(n-1) .\end{cases}
$$

In other words

$$
\operatorname{cohdim}_{\mathbb{Z} / 2 \mathbb{Z}} B\left(\mathbb{R}^{n}, k\right)=(k-\alpha(k)) \cdot(n-1) .
$$

Proof For $k=2$ this follows from [14, Section 4,4.2] with an elementary proof on page 144f. For arbitrary $k \geq 2$ this follows from the theorem in [23, page 31] once one is familiar with Vassiliev's cell decomposition. We do not want to repeat this construction but give some hints for the reader who wants to get acquainted with Vassiliev's notation and the labeling trees $\Gamma_{K_{i}}$ that occur in his theorem: For the case of $H^{*}\left(B\left(\mathbb{R}^{n}, k\right)\right.$ ) (which is $H^{*}\left(\mathbb{R}^{n}(k)\right)=H^{*}\left(\mathbb{R}^{n}(m)\right)$ in Vassiliev's notation, hence $k=m$ ) such a tree has vertices concentrated on $n+1$ horizontal lines and branches from top to bottom. A typical example is given in [23, Figure 12, page 31]. The depth of such a tree is the number of the highest horizontal line beneath which no more branchings exist, see [23, Figure 11, page 30]. The branching condition (page 30 , bottom) implies that the number of vertices on each horizontal line is a power of two and the tree $\Gamma_{K_{i}}$ has $2^{\left|K_{i}\right|}$ vertices on the bottom line. The $m$ in $\left[K_{1}, \ldots, K_{l} ; m\right]$ stands for $m-2^{\left|K_{1}\right|}-\cdots-2^{\left|K_{l}\right|}$ copies of the unique tree $\Gamma_{0}$ that is just a vertical chain of edges without any branching. This means that if we add up the number of vertices on the bottom horizontal lines over all the trees of the collection $\left[K_{1}, \ldots, K_{l} ; m\right]$, the sum is $m$. We are looking for an additive generator of maximal degree, which means that we are looking for a collection $\left[K_{1}, \ldots, K_{l} ; m\right]$ where the sum of the edges of all the trees is minimal. This is because $H^{*}\left(B\left(\mathbb{R}^{n}, k\right)\right)$ is obtained via Poincare duality from the space $B\left(\mathbb{R}^{n}, k\right)_{\infty}$ whose cells correspond to trees, its dimensions correspond to the number of edges. From the fact that on each bottom line the number of vertices is a power of two and their sum is $k=m$, it follows that a collection $\left[K_{1}, \ldots, K_{l} ; m\right]$ consists of at least $\alpha(m)=\alpha(k)$ trees. For $k=2^{l_{1}}+\cdots+2^{l_{\alpha(k)}}$ such a collection of trees with the minimal number of edges is given in Figure 1. 


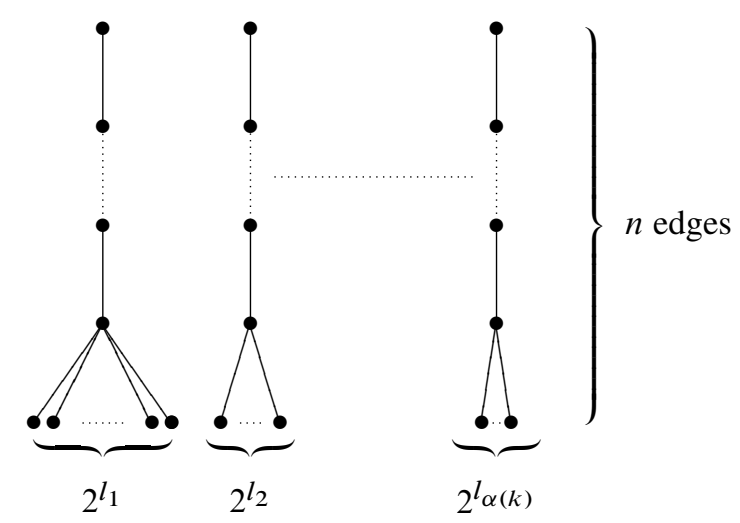

Figure 1: A collection of trees representing an additive generator in $H^{(n-1)(k-\alpha(k))}\left(B\left(\mathbb{R}^{n}, k\right), \mathbb{Z} / 2 \mathbb{Z}\right)$.

This collection has $k+(n-1) \cdot \alpha(k)$ edges and hence represents a generator of $H^{*}\left(B\left(\mathbb{R}^{n}, k\right)\right)$ in degree $n k-(k+(n-1) \cdot \alpha(k))=(n-1) \cdot(k-\alpha(k))$. More details, pictures and examples are also given in [20, pages 25-36]. In particular, see Satz 2.9 on page 34 .

There is also a statement for $\mathbb{Z} / p \mathbb{Z}$-coefficients available in literature, which we shall exploit later. It goes back to Fred Cohen $[4 ; 6]$ and was proved anew and stated explicitly by Ossa (see [19, Proposition 3.4] and the following remark for $p=3$ ):

Proposition 3.6 If $p$ is an odd prime, then

$$
H^{(p-1)(n-1)}\left(\Sigma_{p} ; \mathbb{Z} / p \mathbb{Z}\right) \longrightarrow H^{(p-1)(n-1)}\left(B\left(\mathbb{R}^{n}, p\right) ; \mathbb{Z} / p \mathbb{Z}\right)
$$

is an isomorphism.

The reader interested in cohomological dimensions of configuration spaces should have a look at Kallel [17].

\section{Calculations and proofs}

We begin with the partly special arguments for the computation of $\operatorname{cat}\left(F\left(\mathbb{R}^{n}, k\right)\right)$ and $\operatorname{cat}\left(B\left(\mathbb{R}^{n}, k\right)\right)$ in case $n$ or $k$ is less or equal to 2 .

$k=1 \quad$ Clearly, $\operatorname{cat}\left(F\left(\mathbb{R}^{n}, 1\right)\right)=\operatorname{cat}\left(B\left(\mathbb{R}^{n}, 1\right)\right)=\operatorname{cat}\left(\mathbb{R}^{n}\right)=0$.

$n=1$ If $n=1$, then $F(\mathbb{R}, k)$ has $k$ ! contractible components and $B(\mathbb{R}, k)$ is contractible, hence $\operatorname{cat}(F(\mathbb{R}, k))=k !-1$ and $\operatorname{cat}(B(\mathbb{R}, k))=0$. 
$k=2$ Next, since $\mathbb{R}^{n}$ is a topological group we have $F\left(\mathbb{R}^{n}, 2\right) \cong \mathbb{R}^{n} \times F\left(\mathbb{R}^{n}-\{0\}, 1\right) \simeq$ $\mathbb{S}^{n-1}[6]$ and hence $\operatorname{cat}\left(F\left(\mathbb{R}^{n}, 2\right)\right)=1$ for all $n \geq 1$. In the unordered case we use the homotopy equivalence $B\left(\mathbb{R}^{n}, 2\right) \simeq \mathbb{R} P^{n-1}[6]$ which $\operatorname{implies} \operatorname{cat}\left(B\left(\mathbb{R}^{n}, 2\right)\right)=$ $\operatorname{cat}\left(\mathbb{R} P^{n-1}\right)=n-1$, bounded above by the dimension and below by the mod 2 cuplength.

$n=2$ We have $k-1=\operatorname{cup}_{\mathbb{Z}}\left(F\left(\mathbb{R}^{2}, k\right)\right) \leq \operatorname{cat}\left(F\left(\mathbb{R}^{2}, k\right)\right) \leq \operatorname{cat}\left(B\left(\mathbb{R}^{2}, k\right)\right) \leq k-1$ resulting from Corollary 3.1, Proposition 2.1 (4) and (2) and the more general Lemma 4.1.

Lemma 4.1 For all $n$ and $k$ we have $\operatorname{cat}\left(B\left(\mathbb{R}^{n}, k\right)\right) \leq(k-1) \cdot(n-1)$.

Proof We can assume $n \geq 2$. Then the lemma follows from Proposition 3.3 and Lemma 1.6.

Proof of Theorem 1.2 We now can assume $n, k \geq 3$. Then $k-1=\operatorname{cup}_{\mathbb{Z}}\left(F\left(\mathbb{R}^{n}, k\right)\right) \leq$ $\operatorname{cat}\left(F\left(\mathbb{R}^{n}, k\right)\right) \leq k-1$ follows from 3.1, 2.1(4) and (5). Remember that $F\left(\mathbb{R}^{n}, k\right)$ is $(n-2)$-connected.

Proof of Theorem 1.4 It follows from Proposition 3.4 together with Proposition 2.2, that the degree of each non-zero cohomology class in $H^{*}\left(B\left(\mathbb{R}^{n}, k\right) ; \mathbb{Z} / 2 \mathbb{Z}\right)$ gives a lower bound for $\operatorname{secat}\left(\pi_{k}^{n}\right)$. Hence by Corollary 3.5 and the previous Lemma 4.1 we have $(k-\alpha(k)) \cdot(n-1)=\operatorname{cohdim}_{\mathbb{Z} / 2 \mathbb{Z}}\left(B\left(\mathbb{R}^{n}, k\right)\right) \leq \operatorname{secat}\left(\pi_{k}^{n}\right) \leq \operatorname{cat}\left(B\left(\mathbb{R}^{n}, k\right)\right) \leq$ $(k-1) \cdot(n-1)$. Here we use 2.1(3) and Lemma 4.1 again.

Proof of Theorem 1.1 For notational convenience we just formulate the proof for $r=$ 1. The other cases are similar. We first show $\operatorname{secat}\left(\pi_{k}^{n}\right)=\operatorname{cat}\left(B\left(\mathbb{R}^{n}, k\right) \hookrightarrow B\left(\mathbb{R}^{n+1}, k\right)\right)$ and denote the inclusion by $f$ as in the fundamental diagram (1). Consider $\sec a t\left(\pi_{k}^{n}\right)$ as the category of a classifying map $B\left(\mathbb{R}^{n}, k\right) \rightarrow B\left(\mathbb{R}^{\infty}, k\right)$. The classifying map of $\pi_{k}^{n}$ factors through $f$, hence $\operatorname{secat}\left(\pi_{k}^{n}\right) \leq \operatorname{cat}(f)$.

Now consider the left square in diagram (1). Given a subset $A \subset B\left(\mathbb{R}^{n}, k\right)$ over which $\pi_{k}^{n}$ is trivial, we can factor $\left.f\right|_{A}$ as $\pi_{k}^{n+1} \circ \tilde{f} \circ s$ where $s$ is a local section for $\pi_{k}^{n}$ over $A$. Now we observe that $F\left(\mathbb{R}^{n}, k\right)$ is contractible in $F\left(\mathbb{R}^{n+1}, k\right)$ : Remember that a point in $F\left(\mathbb{R}^{n}, k\right)$ is a $k$-tuple in $\mathbb{R}^{n} \cong \mathbb{R}^{n} \times\{0\} \subset \mathbb{R}^{n+1}$. First move the $k$ points of such a tuple linearly by varying only their last coordinates such that in the end the $i^{\text {th }}$ point lies in $\mathbb{R}^{n} \times\{i\}$. Then continue moving the $i^{\text {th }}$ point linearly to $(0, \ldots, 0, i)$. The fact that $F\left(\mathbb{R}^{n}, k\right)$ is contractible in $F\left(\mathbb{R}^{n+1}, k\right)$ implies that the restriction of $f$ to $A$ is nullhomotopic. This shows $\operatorname{cat}(f) \leq \operatorname{secat}\left(\pi_{k}^{n}\right)$. 
The second equality in Theorem 1.1 can be proved quite elementary by pulling back and extending categorical covers. Remember that a point in $U_{i} \subset B\left(\mathbb{R}^{n}, k\right)$ is a $k-$ element subset of $\mathbb{R}^{n} \cong \mathbb{R}^{n} \times\{0\}$. If $U_{i}$ is open and contractible, extend it to an open contractible subset of $B\left(\mathbb{R}^{n+1}, k\right)$ by simply letting vary the elements of its points in their $(n+1)^{\text {st }}$ coordinates within an open interval, say $(-1,1)$. Alternatively one can apply the next lemma and the fact that the spaces $B\left(\mathbb{R}^{n}, k\right)$ are normal (as $\mathrm{CW}$-complexes) and absolute neighborhood retracts (ANR's) as retracts of some open subset of some $\mathbb{R}^{N}$. For a definition of ANR see the appendix of [9] and Warner [25] for a more detailed introduction.

Lemma 4.2 If $i: A \hookrightarrow B$ is a closed inclusion between normal $A N R$ 's, then $\mathrm{cat}_{B}(A)=$ $\operatorname{cat}(i)$.

Proof Given a categorical cover for $\operatorname{cat}_{B}(A)$, inverse images under $i$ give a categorical cover for $\operatorname{cat}(i)$, hence $\operatorname{cat}(i) \leq \operatorname{cat}_{B}(A)$. Vice versa, given a categorical cover $U_{0}, \cdots, U_{k}$ for cat $(i)$, we can pass to an open refinement $V_{0}, \cdots, V_{k}$ with $V_{i} \subset \bar{V}_{i} \subset U_{i}$ since $A$ is normal [9, Theorem A.1]. Hence $A$ can be covered with $k+1$ sets, each closed and contractible in $B$. In [9] this fact is denoted by $\operatorname{cat}^{c l}{ }_{B}(A) \leq k$ and under the hypothesis that $B$ is a normal ANR and $A \subset B$ is closed, [9, Theorem 1.10 ] says that $\operatorname{cat}^{c l} B(A)=\operatorname{cat}_{B}(A)$. Hence we have $\operatorname{cat}_{B}(A) \leq \operatorname{cat}(i)$.

Proof of Theorem 1.5 The statement for $k$ a power of 2 is a corollary to Theorem 1.4. If $k=p$ is an odd prime, we combine Proposition 3.6 with the group cohomology $H^{*}\left(\Sigma_{p} ; \mathbb{Z} / p \mathbb{Z}\right) \cong \mathbb{Z} / p \mathbb{Z}[\alpha] \otimes \Lambda_{p}(\beta)$ where $\alpha$ is a polynomial generator in degree $2(p-1)$ and $\beta$ is an exterior generator in degree $2(p-1)-1$. This can be derived from Adem and Milgram [1, VI.1.4,1.6, III.2.9] or see Ossa [19] for the statement. Hence $H^{(p-1)(n-1)}\left(\Sigma_{p} ; \mathbb{Z} / p \mathbb{Z}\right) \neq 0$ if $n$ is odd. Now we can argue as in the proof of Theorem 1.4 and obtain

$$
\operatorname{secat}\left(\pi_{p}^{n}\right) \geq \begin{cases}(p-1) \cdot(n-1) & \text { if } n \text { is odd } \\ (p-1) \cdot(n-2) & \text { if } n \text { is even }\end{cases}
$$

The inequality for even $n$ is a consequence of the statement for odd $n$, since

$$
H^{*}\left(\Sigma_{p} ; \mathbb{Z} / p \mathbb{Z}\right) \rightarrow H^{*}\left(B\left(\mathbb{R}^{n-1}, p\right) ; \mathbb{Z} / p \mathbb{Z}\right)
$$

factors via $H^{*}\left(B\left(\mathbb{R}^{n}, p\right) ; \mathbb{Z} / p \mathbb{Z}\right)$. The improvement for $k=3$ then follows from the next lemma and the statement $\operatorname{cat}\left(B\left(\mathbb{R}^{2}, k\right)\right)=(k-1)$ was already shown at the beginning of this section.

Lemma 4.3 For all $n$ we have

$$
\operatorname{secat}\left(\pi_{3}^{n+1}\right) \leq \operatorname{secat}\left(\pi_{3}^{n}\right)+2
$$


Proof We partition $B\left(\mathbb{R}^{n+1}, 3\right)=\coprod_{k=1}^{3} V_{k}\left(\mathbb{R}^{n+1}\right)$, where $V_{k}\left(\mathbb{R}^{n+1}\right)$ is the submanifold of all 3-configurations in $\mathbb{R}^{n+1}$ whose image under the perpendicular projection onto $\mathbb{R}^{n} \cong \mathbb{R}^{n} \times\{0\} \subset \mathbb{R}^{n+1}$ consists of exactly $k$ points. $V_{1}\left(\mathbb{R}^{n+1}\right)$ is obiously contractible. The space $V_{2}\left(\mathbb{R}^{n+1}\right)$ is not necessarily contractible but it is contractible whithin $B\left(\mathbb{R}^{n+1}, 3\right)$. This can be seen by an argument similar to the one that we used in order to show that $F\left(\mathbb{R}^{n}, k\right)$ is contractible within $F\left(\mathbb{R}^{n+1}, k\right)$. The space $V_{2}\left(\mathbb{R}^{n}\right)$ can be contracted within $B\left(\mathbb{R}^{n+1}, 3\right)$ in the following way: Move the three points (making up a point in $V_{2}\left(\mathbb{R}^{n}\right)$ ) linearly by varying only their last coordinates to obtain a three-element subset of $\mathbb{R}^{n+1}$ of the form $\{(x,-1),(\tilde{x}, 0),(\tilde{x}, 1)\}$ (here $\left.x, \tilde{x} \in \mathbb{R}^{n}\right)$, then move this linearly to $\{(0,0,-1),(0,0,0),(0,0,1)\}$. Furthermore cat $_{B\left(\mathbb{R}^{n+1}, 3\right)}\left(V_{3}\left(\mathbb{R}^{n+1}\right)\right) \leq \operatorname{cat}\left(B\left(\mathbb{R}^{n}, 3\right)\right)$, since $V_{3}\left(\mathbb{R}^{n+1}\right) \subset B\left(\mathbb{R}^{n+1}, 3\right)$ is open and retractible to $B\left(\mathbb{R}^{n}, 3\right)$. Now we should pass to tubular neighborhoods $U_{1}, U_{2}$ of $V_{1}, V_{2}$ in order to have open contractible sets available. We obtain

$$
\begin{aligned}
\operatorname{secat}\left(\pi_{3}^{n+1}\right) & \leq \operatorname{cat}\left(B\left(\mathbb{R}^{n+1}, 3\right)\right) \\
& =\operatorname{cat}\left(\coprod_{k=1}^{3} V_{k}\left(\mathbb{R}^{n+1}\right)\right) \\
& =\operatorname{cat}_{B\left(\mathbb{R}^{n+1,3)}\right.}\left(U_{1} \cup U_{2} \cup V_{3}\right) \\
& \leq \operatorname{cat}_{B\left(\mathbb{R}^{n+1}, 3\right)}\left(V_{3}\right)+\operatorname{cat}_{B\left(\mathbb{R}^{n+1,3}\right)}\left(U_{2}\right)+\operatorname{cat}_{B\left(\mathbb{R}^{n+1,3}\right)}\left(U_{1}\right)+2 \\
& \leq \operatorname{cat}_{B\left(\mathbb{R}^{n+1}, 3\right)}\left(B\left(\mathbb{R}^{n}, 3\right)\right)+2 \\
& =\operatorname{secat}\left(\pi_{3}^{n}\right)+2 .
\end{aligned}
$$

The last inequality follows from Theorem 1.1 with $r=1$.

\section{References}

[1] A Adem, R J Milgram, Cohomology of finite groups, second edition, Grundlehren series 309, Springer, Berlin (2004) MR2035696

[2] G Arone, A note on the homology of $\Sigma_{n}$, the Schwartz genus, and solving polynomial equations, from: "An alpine anthology of homotopy theory", Contemp. Math. 399, Amer. Math. Soc., Providence, RI (2006) 1-10 MR2222501

[3] I Berstein, On the Lusternik-Schnirelmann category of Grassmannians, Math. Proc. Cambridge Philos. Soc. 79 (1976) 129-134 MR0400212

[4] F Cohen, Cohomology of braid spaces, Bull. Amer. Math. Soc. 79 (1973) 763-766 MR0321074

[5] F R Cohen, On configuration spaces, their homology, and Lie algebras, J. Pure Appl. Algebra 100 (1995) 19-42 MR1344842 
[6] F R Cohen, T J Lada, J P May, The homology of iterated loop spaces, Lecture Notes in Mathematics 533, Springer (1976) MR0436146

[7] F R Cohen, L R Taylor, Computations of Gel'fand-Fuks cohomology, the cohomology of function spaces, and the cohomology of configuration spaces, from: "Geometric applications of homotopy theory (Proc. Conf., Evanston, Illinois, 1977), I", Lecture Notes in Math. 657, Springer, Berlin (1978) 106-143 MR513543

[8] R L Cohen, The immersion conjecture for differentiable manifolds, Ann. of Math. (2) 122 (1985) 237-328 MR808220

[9] O Cornea, G Lupton, J Oprea, D Tanré, Lusternik-Schnirelmann category, Mathematical Surveys and Monographs 103, American Mathematical Society, Providence, RI (2003) MR1990857

[10] C De Concini, C Procesi, M Salvetti, On the equation of degree 6, Comment. Math. Helv. 79 (2004) 605-617 MR2081728

[11] E R Fadell, S Y Husseini, Geometry and topology of configuration spaces, Springer Monographs in Mathematics, Springer, Berlin (2001) MR1802644

[12] E Fadell, L Neuwirth, Configuration spaces, Math. Scand. 10 (1962) 111-118 MR0141126

[13] M Farber, S Tabachnikov, S Yuzvinsky, Topological robotics: motion planning in projective spaces, Int. Math. Res. Not. (2003) 1853-1870 MR1988783

[14] D B Fuks, Cohomology of the braid group mod 2, Funkcional. Anal. i Priložen. 4 (1970) 62-73 MR0274463 English translation in Funct. Anal. Appl. 4 (1970) 143-151

[15] J González, Topological robotics in lens spaces, Math. Proc. Cambridge Philos. Soc. 139 (2005) 469-485 MR2177172

[16] A Hatcher, Algebraic topology, Cambridge University Press (2002) MR1867354

[17] S Kallel, Symmetric Products and Configuration Spaces, from: "Groups, homotopy and configuration spaces (Tokyo 2005)", (N Iwase, T Kohno, R Levi, D Tamaki, J Wu, editors), Geom. Topol. Monogr. 13 (2008) 499-XXX

[18] L Lusternik, L Schnirelmann, Méthodes topologiques dans les problèmes variationnels, Hermann, Paris (1934)

[19] E Ossa, On the cohomology of configuration spaces, from: "Algebraic topology: new trends in localization and periodicity (Sant Feliu de Guíxols, 1994)", Progr. Math. 136, Birkhäuser, Basel (1996) 353-361 MR1397743

[20] F Roth, Zur Lusternik-Schnirelmann-Kategorie Euklidischer Konfigurationsräume, Diplomarbeit, Universität Bonn Available at http://www.math.uni-hamburg.de/ home/roth/

[21] S Smale, On the topology of algorithms. I, J. Complexity 3 (1987) 81-89 MR907191 
[22] A S Švarc, The genus of a fiber space, Dokl. Akad. Nauk SSSR (N.S.) 119 (1958) 219-222 MR0102812 English translation in AMS Transl. (2) 55 (1966) 49-140

[23] V A Vassiliev, Complements of discriminants of smooth maps: topology and applications, Translations of Mathematical Monographs 98, Amer. Math. Soc. (1992) MR1168473 Translated from the Russian by B Goldfarb

[24] V A Vassillev, Cohomology of braid groups and the complexity of algorithms, Funktsional. Anal. i Prilozhen. 22 (1988) 15-24, 96 MR961758

[25] G Warner, Topics in Topology and Homotopy Theory, Hopf archive. Available at http://hopf.math.purdue.edu/

Fachbereich Mathematik der Universität Hamburg, SPAZ, Bundesstrasse 55

20146 Hamburg, Germany

roth@math.uni-hamburg.de

http://www . math. uni-hamburg.de/home/roth/

Received: 31 May 2006 Revised: 22 March 2007 
